\title{
In situ electrical characterization of dielectric thin films directly exposed to plasma vacuum-ultraviolet radiation
}

\author{
C. Cismaru a) and J. L. Shohet \\ Center for Plasma-Aided Manufacturing and Department of Electrical \& Computer Engineering, \\ University of Wisconsin-Madison, Madison, Wisconsin 53706
}

(Received 5 January 2000; accepted for publication 8 May 2000)

\begin{abstract}
In this article we report a method for in situ electrical characterization of dielectric thin films under direct exposure to plasma in an electron-cyclotron-resonance etcher. This method is based on the development of a special test structure that allows for the measurement of the influence of plasma vacuum-ultraviolet (VUV) radiation on the electrical conductivity of thin dielectric layers. Results show that the measured conductivity of $\mathrm{SiO}_{2}$ layers temporarily increases during exposure to argon and oxygen plasmas, with controlled VUV emission. Based on the measurements made through this method, a model of the VUV-induced conductivity of $\mathrm{SiO}_{2}$ is developed. These measurements are very important for plasma processing of semiconductor devices, because the temporary increase in the conductivity of these layers upon exposure to processing plasmas can decrease the plasma-induced charging of these dielectric layers depending on the intensity of the plasma VUV emission. This can have an impact on the properties and reliability of processed devices. (C) 2000 American Institute of Physics. [S0021-8979(00)03016-4]
\end{abstract}

\section{INTRODUCTION}

Today, plasma processing accounts for over $30 \%$ of the total processing steps involved in very large scale integration (VLSI) and ultra large scale integration (ULSI) semiconductor device manufacturing. ${ }^{1}$ During plasma processing, the electrical characteristics (e.g., conductivity) of dielectrics that are directly exposed to the plasma are influenced by plasma-emitted vacuum-ultraviolet (VUV) radiation. ${ }^{2-4}$ It was determined that most high-density plasmas emit radiation in the VUV energy band between 4 and $30 \mathrm{eV}$, with most of the radiation above $9 \mathrm{eV},{ }^{5}$ the latter of which is approximately the energy band gap of $\mathrm{SiO}_{2}$. This radiation can be absorbed in exposed dielectric layers, and it results in the generation of electron-hole pairs. ${ }^{6}$ The extra charge carriers can induce a temporary increase in the conductivity of these layers, which will have an impact on the properties and reliability of processed devices.

Although this is an issue of great importance for semiconductor device manufacturing, there are no studies to date that report monitoring of the changes in the electrical characteristics of dielectrics in situ, during exposure to plasma. The reason is that it is impossible to measure voltages across thin dielectric layers and the electric currents passing through them directly. Such measurements require metallic electrodes above and below the thin dielectric layer, which then block the plasma VUV radiation, rendering the measurements irrelevant. To overcome this problem, thin (100 $\AA$ ) aluminum layers were used in some studies ${ }^{7}$ as measuring electrodes, which are semitransparent at this thickness to VUV radiation in the range of 15 to $70 \mathrm{eV}$. However, these measurements were not performed in situ and, in addition, only the effects of plasma VUV radiation on the electrical

${ }^{a)}$ Currently with Conexant Systems, Inc., Newport Beach, CA. characteristics of $\mathrm{SiO}_{2}$ for photons between 15 and $70 \mathrm{eV}$ were determined, while most of the plasma VUV emission is between 9 and $15 \mathrm{eV} .^{5}$ In addition, any temporary effects that could result in in situ changes in the electrical characteristics of the dielectric layers were not measured.

The purpose of this article is to demonstrate a method for the in situ measurement of the changes in the electrical characteristics (e.g., electric conductivity) of thin dielectric layers. Based on this method, measurements of the electrical conductivity of thin (less than $3000 \AA$ ) $\mathrm{SiO}_{2}$ layers during exposure to argon and oxygen plasma in an electroncyclotron-resonance (ECR) etcher will be demonstrated.

\section{EXPERIMENTAL METHOD}

The method was implemented in the ECR plasmaetching system shown in Fig. 1. The system incorporated a $1.5 \mathrm{~kW}$ microwave plasma source and a pair of magnets arranged in a vertical magnetic-mirror configuration. ${ }^{8}$ The test structures necessary for these measurements were placed on the wafer stage that is located $19 \mathrm{~cm}$ below the electroncyclotron-resonance region. For this work, the neutral pressure and microwave power were held constant at $2 \mathrm{mTorr}$ and $1000 \mathrm{~W}$, respectively. Either argon or oxygen was used as the feed gas. Under these conditions, plasmas with densities in the $10^{11}$ to $10^{12} \mathrm{~cm}^{-3}$ range were obtained. No wafer bias was applied.

The method we report here is based on the development of a special test structure, which allows for the in situ measurement of the $J-E$ (current density vs electric field) curves for thin dielectric layers that are directly exposed to the plasma. The test structure and its required electrical circuitry are shown in Fig. 2. We will show that this test structure and its associated measurement procedure allow us to obtain the current-voltage relationship between the current passing 


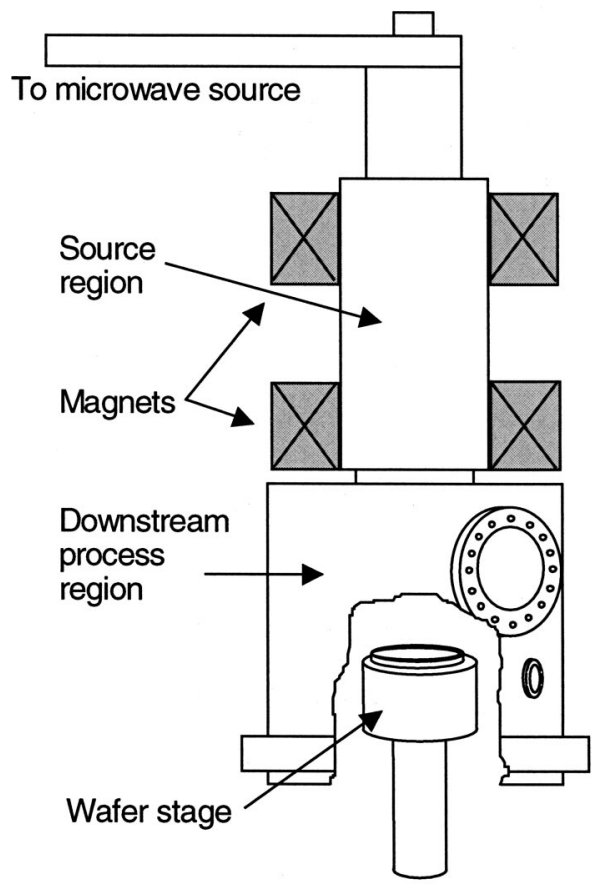

FIG. 1. Schematic of the ECR plasma etching system.

through the thin oxide layer and the voltage drop across it, even though we do not make a direct measurement of this voltage drop.

The test structure development procedure is as follows. A $5.8 \times 5.8 \mathrm{~mm} \mathrm{Al}$ pad is deposited on top of a $1-\mu \mathrm{m}$ thick thermally grown oxide on a $\mathrm{Si}$ wafer $(n$-type, $1 \Omega \mathrm{cm})$. The $\mathrm{Al}$ pad is covered with a thin layer of PECVD-deposited (plasma-enhanced-chemical-vapor-deposition) $\mathrm{SiO}_{2}$ of varying thicknesses, which is the layer to be tested. It is labeled "covered pad" in Fig. 2. In addition, a second pad, with identical shape and area, is located $6 \mathrm{~mm}$ away from the first, but is not covered by oxide (labeled "uncovered pad" in Fig. 2). For the purpose of this article, three separate sets of test structures of this kind were built (on separate wafers), with PECVD oxide thicknesses (as measured after deposition) of 570,690 , and $2925 \AA$. The nonuniformity of each thickness was less than $\pm 5 \%$ across the surface of the wafers.
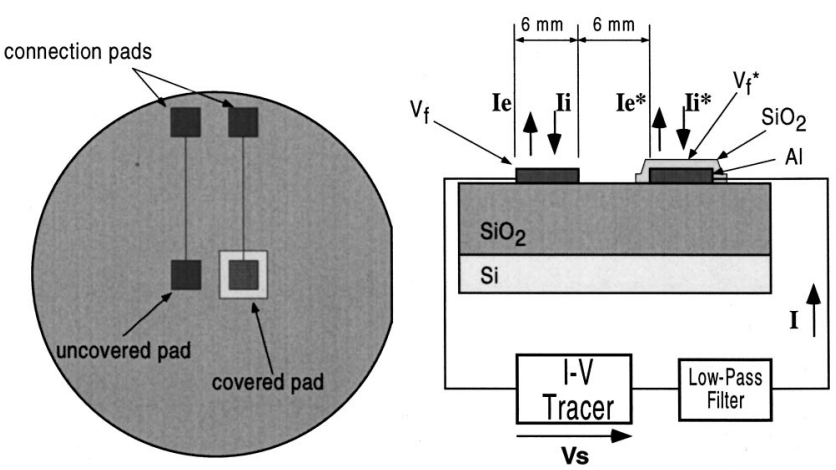

FIG. 2. Diagram of the test structure and the associated measurement circuitry.

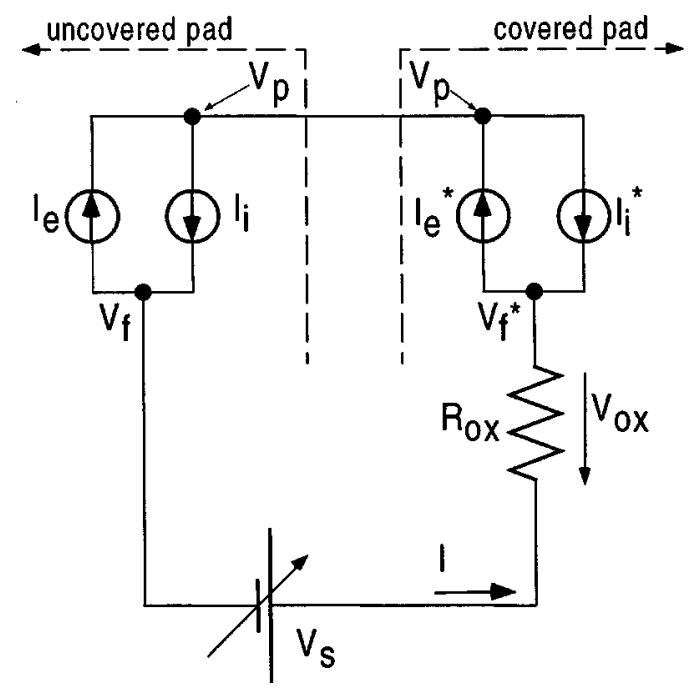

FIG. 3. Equivalent electric circuit for the measurement setup in Fig. 2.

\section{THEORETICAL MODELING}

The equivalent electrical circuit of the setup shown in Fig. 2 is displayed in Fig. 3. In this circuit, the thin oxide covering the pad has been modeled as a nonlinear resistor $R_{\text {ox }}$, with a voltage drop $V_{\text {ox }}$ across it. The floating potential on the surface of the oxide with respect to ground is $V_{f}^{*}$. Similarly, the floating potential with respect to ground on the uncovered pad is $V_{f}$. $V_{p}$ is the plasma potential above the two pads measured with respect to ground, and it is assumed uniform and constant during the measurement. Subsequent measurements will show that the current flowing through the circuit in Fig. 3 is small (less than the ion current arriving at the pads) and thus, the previous assumption is valid. $V_{s}$ is the externally applied voltage of the $I-V$ (current vs voltage) tracer (which can be varied between -30 and $+30 \mathrm{~V}$ ). $I_{i}^{*}$ and $I_{i}$ are the ion currents impinging on the surface of the covered and the uncovered pads, respectively. Similarly, $I_{e}^{*}$ and $I_{e}$ are the corresponding electron currents, which are represented as nonlinear voltage-dependent current sources by considering a Maxwellian distribution of electrons ${ }^{9}$ (an assumption under these plasma conditions), such that

$$
\begin{aligned}
& I_{e}^{*}=I_{\mathrm{sat}} e^{\left(V_{j}^{*}-V_{p}\right) / T_{e}} \quad[\mathrm{~A}], \\
& I_{e}=I_{\mathrm{sat}} e^{\left(V_{f}-V_{p}\right) / T_{e}} \quad[\mathrm{~A}],
\end{aligned}
$$

where

$$
I_{\text {sat }}=\frac{1}{4} A e n v_{e} \quad[\mathrm{~A}],
$$

where $v_{e}$ is the average electron velocity, ${ }^{10} A$ is the pad area, and $n$ is the plasma density. Temperatures are in units of $\mathrm{eV}$. We assume that both pads have the same area. The potentials $V_{f}$ and $V_{f}^{*}$ can be determined by balancing all the currents in the circuit. For the equivalent electrical circuit in Fig. 3, we can write the following equations:

$$
\begin{aligned}
& I_{e}^{*}=I+I_{i}^{*}, \\
& I_{i}-I_{e}=I, \\
& V_{s}-V_{\mathrm{ox}}-V_{f}^{*}+V_{f}=0 .
\end{aligned}
$$




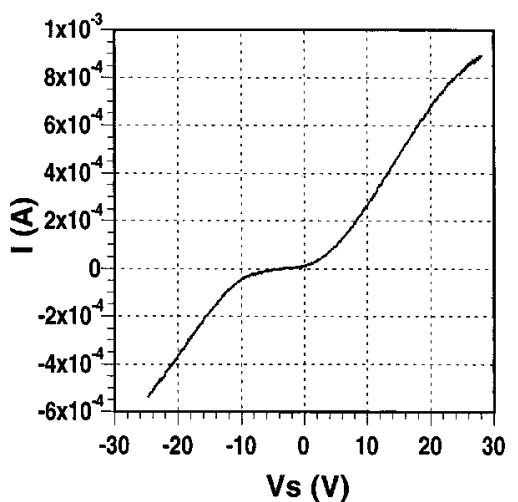

FIG. 4. $I-V$ measurement on the $690 \AA$ oxide-covered $\mathrm{Al}$ pad for an $\mathrm{O}_{2}$ plasma at $1000 \mathrm{~W}$ microwave power and 2 mTorr neutral pressure.

In addition, because the pads have the same area, we assume that the ion currents arriving at the surface of both the covered and the uncovered pads are equal and defined by the Bohm criterion and the ion flux continuity requirement for collisionless plasma sheaths ${ }^{10}$ as shown in Eq. (7)

$$
I_{i}=I_{i}^{*}=0.6 \text { en }_{B} \quad[\mathrm{~A}],
$$

where $A$ is the area of the pads, $e$ is the electronic charge, $n$ is the plasma density, and $u_{B}$ is the ion Bohm velocity. ${ }^{10}$ Thus, under these assumptions, we can add Eqs. (4) and (5) to give

$$
2 I=I_{e}^{*}-I_{e} .
$$

If we solve Eq. (6) for $V_{f}^{*}$ and we substitute it into Eq. (1), we find

$$
I_{e}^{*}=I_{e} e^{\left(V_{s}-V_{\mathrm{ox}}\right) / T_{e}} .
$$

Substituting $I_{e}^{*}$ from Eq. (8) into Eq. (9), and taking the log of both sides, we can then find a relationship between $V_{\text {ox }}$ and $I$ as

$$
V_{\mathrm{ox}}=V_{s}-T_{e} \ln \left(1+\frac{2 I}{I_{e}}\right) .
$$

Therefore, we obtain the $J_{\text {ox }}$ vs $E_{\text {ox }}$ relationship through the dielectric layer by making the following measurements. First, a measurement of the current $I$ as a function of $V_{s}$ is made, by varying $V_{s}$ between -10 and $10 \mathrm{~V}$ in the setup shown in Figs. 1 and 2. One example of this measurement is shown in Fig. 4. Next, a Langmuir probe measurement ${ }^{11}$ using the uncovered pad is made so as to extract the electron temperature $T_{e}$ and the electron current $I_{e}$. The electron current is extracted as a function of the pad's floating potential $V_{f}$. An example of the Langmuir probe measurement is given in Fig. 5. To relate the electron current $I_{e}$ to the source voltage $V_{s}$ as in Eq. (10), a measurement of $V_{f}$ during the sweep of $V_{s}$ is also made. The measurement of $V_{f}$ vs $V_{s}$ is made by using a high-voltage probe, with an impedance of $100 \mathrm{M} \Omega$, connected to the uncovered $\mathrm{Al}$ pad. This gives us all the terms on the right-hand side of Eq. (10) except for the current $I$ and therefore we can determine the $I-V$ relationship for the oxide layer and the $J_{\mathrm{ox}}-E_{\mathrm{ox}}$ relationship by subsequently dividing the current $I$ by the pad area and the oxide voltage drop $V_{\text {ox }}$ by the oxide thickness.

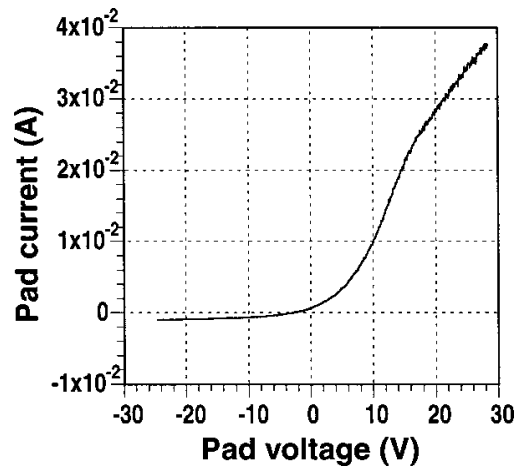

FIG. 5. Langmuir probe measurement on the uncovered $\mathrm{Al}$ pad for an $\mathrm{O}_{2}$ plasma at $1000 \mathrm{~W}$ microwave power and 2 mTorr neutral pressure.

\section{MEASUREMENTS OF THE $\boldsymbol{J}-\boldsymbol{V}$ CHARACTERISTICS OF THIN $\mathrm{SiO}_{2}$ LAYERS DIRECTLY EXPOSED TO PLASMA RADIATION}

Based on the measurement method detailed above, measurements of the $J-V$ characteristics of thin $\mathrm{SiO}_{2}$ layers were made during exposure to argon and oxygen plasmas, at $1000 \mathrm{~W}$ microwave power and 2 mTorr neutral pressure. Examples of these measurements using an argon plasma are provided in Fig. 6 for different oxide thicknesses. Since no significant differences were observed with respect to the polarity of the oxide voltage drop, only unidirectional characteristics are shown. These measurements will be used with the model previously discussed to find the conductivity of the oxide layers.

The measured data can be fitted to a simple photoconductor mode ${ }^{12}$ to determine the conductivity of $\mathrm{SiO}_{2}$ layers under exposure to plasma radiation. This model is defined by the following expression:

$$
J_{\mathrm{ox}}=C \frac{\varphi}{t_{\mathrm{ox}}} E_{\mathrm{ox}}\left[\mathrm{A} / \mathrm{cm}^{2}\right],
$$

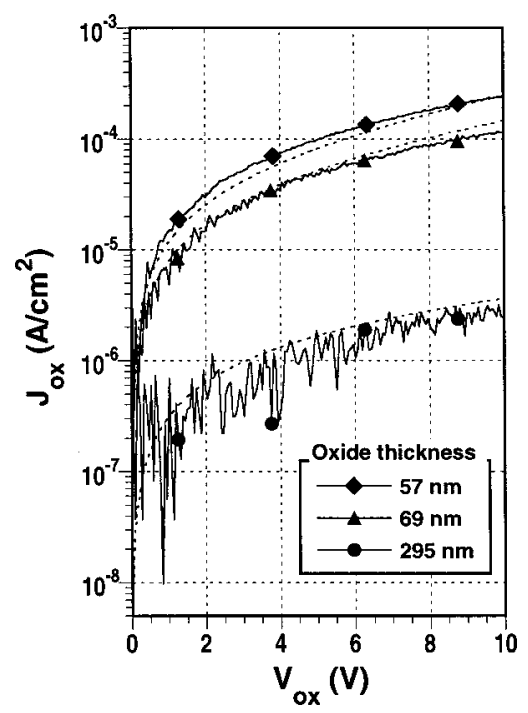

FIG. 6. VUV-induced leakage current density (patterned solid lines) and fitted values (dashed lines) as a function of applied voltage across the oxide layer for different oxide thicknesses. Plasma conditions were Ar, 2 mTorr neutral pressure, and $1000 \mathrm{~W}$ microwave power. 
where $\varphi$ is the ionizing radiation flux with energies higher than the energy bandgap of $\mathrm{SiO}_{2}$ (approximately $9 \mathrm{eV}$ ), $E_{\text {ox }}$ is the electric field across the oxide layer $\left(E_{\mathrm{ox}}=V_{\mathrm{ox}} / t_{\mathrm{ox}}\right), t_{\mathrm{ox}}$ is the oxide thickness, and $C$ is a fitting parameter. The radiation flux was measured previously to be 3.6 $\times 10^{14}$ photons $/ \mathrm{cm}^{2} \mathrm{~s}$ for argon and $5.1 \times 10^{13}$ photons $/ \mathrm{cm}^{2} \mathrm{~s}$ for oxygen, ${ }^{5}$ in the ECR etcher, at 2 mTorr neutral pressure and $1000 \mathrm{~W}$ microwave power.

For the oxide thicknesses used in this work, the high electric field, in connection with the density of impurities and imperfections in the oxide layer, results in an exponential trend of the current density with the applied electric field. It is similar to the current in a regular forward-biased diode ${ }^{13}$ or a metal-semiconductor ohmic contact. ${ }^{14}$ Therefore, Eq. (11) was modified by substituting the expression $E_{\mathrm{ox}} e^{\xi E_{\mathrm{ox}}}$ for the electric field $E_{\mathrm{ox}}$, which adds a second fitting parameter $\xi$. The current density expression is then

$$
J_{\mathrm{ox}}=C \frac{\varphi}{t_{\mathrm{ox}}} E_{\mathrm{ox}} e^{\xi E_{\mathrm{ox}}}
$$

or, in terms of the applied oxide voltage

$$
J_{\mathrm{ox}}=C \frac{\varphi}{t_{\mathrm{ox}}^{2}} V_{\mathrm{ox}} e^{\xi\left(V_{\mathrm{ox}} / t_{\mathrm{ox}}\right)}
$$

This leads to a conductivity that depends on the applied electric field of the form

$$
\sigma_{\mathrm{ox}}=C \frac{\varphi}{t_{\mathrm{ox}}} e^{\xi E_{\mathrm{ox}}}\left[\Omega^{-1} \mathrm{~cm}^{-1}\right] .
$$

The values of the parameters $C$ and $\xi$ were determined for both argon and oxygen plasma conditions, by fitting the experimental data to the model defined in Eq. (13). Figure 6 shows the measured current density across the oxide layer upon exposure to Ar plasma at 2 mTorr neutral pressure and $1000 \mathrm{~W}$ microwave power, for different oxide thicknesses, together with the fitted curves. The standard error of the fitting parameters $C$ and $\xi$ was under $3 \%$. Based on these measurements, the oxide conductivity was determined as shown in Fig. 7. Similar measurements were made for oxygen plasma, under the same neutral pressure and microwave power.

The resulting fitting parameters $C$ and $\xi$ are summarized in Table I. The measurements of the oxide conductivity resulted in values up in the $10^{-10}$ to $10^{-9} \Omega^{-1} \mathrm{~cm}^{-1}$ range during exposure to argon and oxygen plasma, respectively. We believe the higher oxide conductivity induced during exposure to oxygen plasma is due to the stronger emission of this plasma near the $\mathrm{SiO}_{2}$ bandgap energy $(\sim 9 \mathrm{eV})$. In contrast, argon plasma has most of its VUV emission at higher energies $(\sim 12 \mathrm{eV}){ }^{5}$ The lower energy photons emitted by the oxygen plasma will penetrate deeper in the $\mathrm{SiO}_{2}$ layer, ${ }^{15}$ inducing the generation of electron-hole pairs throughout the oxide, while the higher energy photons emitted by the argon plasma will penetrate only a few hundreds of angstroms and will generate electron-hole pairs in this outermost layer only. Therefore, it is expected that the conductivity of oxide layers

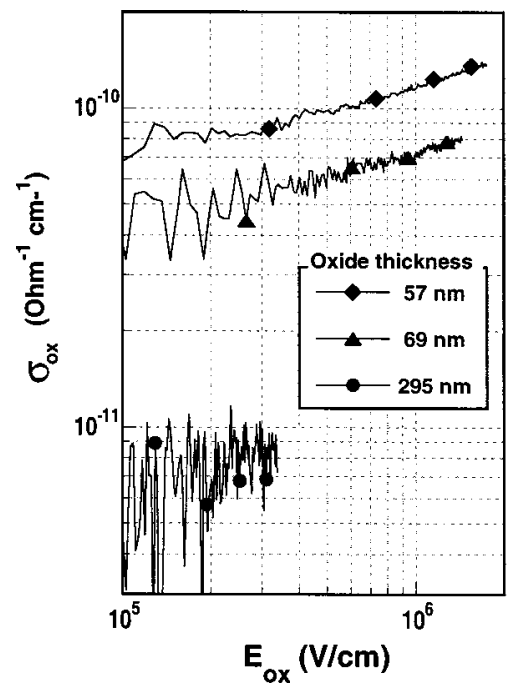

FIG. 7. Measured electrical conductivity of PECVD $\mathrm{SiO}_{2}$ with different oxide thicknesses under exposure to Ar plasma, at 2 mTorr neutral pressure and $1000 \mathrm{~W}$ microwave power.

of more than a few hundreds of angstroms thick will be higher under oxygen plasma exposure than under argon plasma exposure.

The oxide conductivity measured in situ during plasma exposure was compared to the conductivity measured before and after the exposure, under complete darkness. To make these measurements after the plasma exposure, another aluminum layer was added on top of the PECVD oxide layer ("covered pad") by magnetron sputtering, followed by a 20-min metal-anneal step at $425^{\circ} \mathrm{C}$ in forming gas. The $I-V$ curves recorded between the upper and lower $\mathrm{Al}$ layers before and after plasma exposure showed conductivities about three orders of magnitude lower than that measured under plasma exposure. These results brought us to the conclusion that, although the oxide conductivity depends strongly on its quality, it also increases dramatically under exposure to typical high-density plasmas, which have densities and vacuumultraviolet emissions comparable to modern semiconductor processing plasmas. VUV exposure can reduce dielectric charging, especially that induced by electron-shading effects ${ }^{16}$ during plasma etching of high aspect-ratio devices, by providing a safe way to discharge these structures and, thus, minimize charging damage. The enhanced conductivity can also have beneficial effects on the etching properties of $\mathrm{SiO}_{2}$ such as reduction of notching, sidewall bowing, and trenching. ${ }^{17}$

TABLE I. Values of the fitting parameters $C$ and $\xi$ for argon and oxygen plasmas, at $1000 \mathrm{~W}$ microwave power and 2 mTorr neutral pressure.

\begin{tabular}{ccc}
\hline \hline Feed gas & $C\left(\mathrm{~cm}^{2} \mathrm{~s} \Omega^{-1}\right)$ & $\xi\left(\mathrm{cm} \mathrm{V}^{-1}\right)$ \\
\hline $\mathrm{Ar}$ & $9.6 \times 10^{-31}$ & $4.7 \times 10^{-7}$ \\
$\mathrm{O}_{2}$ & $8.9 \times 10^{-30}$ & $8 \times 10^{-7}$ \\
\hline \hline
\end{tabular}




\section{CONCLUSIONS}

In this article we demonstrate a method for the measurement of the electrical characteristics (e.g., electric conductivity) of thin dielectric layers exposed directly to high-density plasma radiation. This method does not require the use of metal electrodes directly attached to the dielectric layer, which block the incoming radiation from impinging on the layer to be studied. Based on this method, in situ measurements of the electrical conductivity of thin $\mathrm{SiO}_{2}$ layers are made during exposure to argon and oxygen plasmas in an ECR etcher. A model of this conductivity as a function of the applied electric field was also provided. Results show that the conductivity of these layers temporarily increases during exposure to processing plasmas. This effect can produce beneficial changes in the plasma-induced charging of these dielectric layers, which can influence the processing properties and the reliability of the processed devices.

\section{ACKNOWLEDGMENTS}

This work was supported in part by the National Science Foundation under Grant No. EEC-8721545 and in part by Texas Instruments.

${ }^{1}$ D. M. Manos and D. L. Flamm, Plasma Etching: An Introduction (Academic, Boston, 1989).
${ }^{2}$ R. A. Gdula, IEEE Trans. Electron Devices 26, 644 (1979).

${ }^{3}$ T. Yunogami, T. Mizutani, K. Suzuki, and S. Nishimatsu, Jpn. J. Appl. Phys. 28, 2172 (1989).

${ }^{4}$ T. Mizutani, in International Symposium on Plasma Process-Induced Damage, edited by K. P. Cheung, M. Nakamura, and C. T. Gabriel (NCCAVS, Sunnyvale, CA, 1996), p. 157.

${ }^{5}$ C. Cismaru and J. L. Shohet, Appl. Phys. Lett. 74, 2599 (1999).

${ }^{6}$ T. P. Ma and P. V. Dressendorfer, Ionizing Radiation Effects in MOS Devices and Circuits (Wiley, New York, 1989).

${ }^{7}$ T. Yunogami, T. Mizutani, K. Tsujimoto, and K. Suzuki, Jpn. J. Appl. Phys. 29, 2269 (1990).

${ }^{8}$ J. B. Friedmann, J. L. Shohet, R. Mau, N. Hershkowitz, S. Bisgaard, S. M. Ma, and J. P. McVittie, IEEE Trans. Semicond. Manuf. 10, 154 (1997).

${ }^{9}$ F. F. Chen, Introduction to Plasma Physics and Controlled Fusion (Plenum, New York, 1983).

${ }^{10}$ M. A. Lieberman and A. J. Lichtenberg, Principles of Plasma Discharges and Materials Processing (Wiley, New York, 1994), pp. 154-167.

${ }^{11}$ N. Hershkowitz, in Plasma Diagnostics: Volume 1, Discharge Parameters and Plasma Chemistry, edited by O. Auciello and D. L. Flamm (Academic, San Diego, 1989), pp. 113-183.

${ }^{12}$ R. H. Bube, Photoconductivity of Solids (Wiley, New York, 1960).

${ }^{13}$ G. W. Neudeck, The PN Junction Diode (Addison-Wesley, Reading, MA, 1989).

${ }^{14}$ R. C. Jaeger, Introduction to Microelectronic Fabrication (AddisonWesley, Reading, MA, 1988).

${ }^{15}$ T. Tatsumi, S. Fukuda, and S. Kadomura, Jpn. J. Appl. Phys. 33, 2175 (1994).

${ }^{16}$ K. Hashimoto, Jpn. J. Appl. Phys. 32, 6109 (1993).

${ }^{17}$ T. Kinoshita, M. Hane, and J. P. McVittie, J. Vac. Sci. Technol. B 14, 560 (1996). 